\title{
On directed 2-factors in digraphs and 2-factors containing perfect matchings in bipartite graphs
}

\author{
Shuya Chiba ${ }^{1 * \dagger} \quad$ Tomoki Yamashita ${ }^{2 \ddagger \S}$ \\ ${ }^{1}$ Applied Mathematics, Faculty of Advanced Science and Technology, Kumamoto University, \\ 2-39-1 Kurokami, Kumamoto 860-8555, Japan \\ ${ }^{2}$ Department of Mathematics, Kindai University, \\ 3-4-1 Kowakae, Higashi-Osaka, Osaka 577-8502, Japan
}

\begin{abstract}
In this paper, we give the following result: If $D$ is a digraph of order $n$, and if $d_{D}^{+}(u)+$ $d_{D}^{-}(v) \geq n$ for every two distinct vertices $u$ and $v$ with $(u, v) \notin A(D)$, then $D$ has a directed 2 -factor with exactly $k$ directed cycles of length at least 3 , where $n \geq 12 k+3$. This result is equivalent to the following result: If $G$ is a balanced bipartite graph of order $2 n$ with partite sets $X$ and $Y$, and if $d_{G}(x)+d_{G}(y) \geq n+2$ for every two vertices $x \in X$ and $y \in Y$ with $x y \notin E(G)$, then for every perfect matching $M, G$ has a 2-factor with exactly $k$ cycles of length at least 6 containing every edge of $M$, where $n \geq 12 k+3$. These results are generalizations of theorems concerning Hamilton cycles due to Woodall (1972) and Las Vergnas (1972), respectively.
\end{abstract}

Keywords: Digraphs, Directed 2-factors, Degree conditions, Perfect matchings, Bipartite graphs

AMS Subject Classification: 05C70, 05C38

\section{Introduction}

We consider only finite graphs. For standard graph-theoretic terminology not explained in this paper, we refer the reader to [5]. Unless stated otherwise, "graph" means a simple undirected graph. Let $G$ be a graph. We denote by $V(G)$ and $E(G)$ the vertex set and the edge set of $G$, respectively. For a vertex $v$ of $G, d_{G}(v)$ denotes the degree of $v$ in $G$. Let $\sigma_{2}(G)$ be the minimum degree sum of two non-adjacent vertices in $G$, i.e., $\sigma_{2}(G)=\min \left\{d_{G}(u)+d_{G}(v)\right.$ : $u, v \in V(G), u \neq v, u v \notin E(G)\}$ if $G$ is not complete; otherwise, $\sigma_{2}(G)=+\infty$.

A graph is said to be hamiltonian if it has a Hamilton cycle, i.e., a cycle containing all the vertices. The following $\sigma_{2}$ condition, due to Ore (1960), is classical and well known in graph theory and there are many results on degree conditions which generalize it (see a survey [9]).

Theorem A (Ore [11]) Let $G$ be a graph of order $n \geq 3$. If $\sigma_{2}(G) \geq n$, then $G$ is hamiltonian.

A 2-factor of a graph is a spanning subgraph in which every component is a cycle, and thus a Hamilton cycle is a 2-factor with exactly 1 cycle. Brandt, Chen, Faudree, Gould and Lesniak (1997) gave the following $\sigma_{2}$ condition for the existence of a 2 -factor with exactly $k$ cycles.

\footnotetext{
${ }^{*}$ This work was supported by JSPS KAKENHI grant 17K05347, 17K05348

${ }^{\dagger}$ E-mail address: schiba@kumamoto-u.ac.jp

‡This work was supported by JSPS KAKENHI grant $16 \mathrm{~K} 05262$

${ }^{\S}$ E-mail address: yamashita@math.kindai.ac.jp
} 
Theorem B (Brandt et al. 4]) Let $k$ be a positive integer, and let $G$ be a graph of order $n \geq 4 k$. If $\sigma_{2}(G) \geq n$, then $G$ has a 2 -factor with exactly $k$ cycles.

Theorem $\mathrm{A}$ is essentially the case $k=1$ of Theorem $\mathrm{B}$, since the result of Theorem $\mathrm{A}$ is easy to see when $n=3$; thus Theorem $\mathrm{B}$ implies Theorem $\mathrm{A}$.

For a digraph $D$, we denote by $A(D)$ the arc set of $D$, and let $d_{D}^{+}(v)$ and $d_{D}^{-}(v)$ be the out-degree and the in-degree of a vertex $v$ in $D$, respectively. A directed Hamilton cycle is a directed cycle containing all the vertices of the digraph, and a directed 2-factor of a digraph is a spanning subdigraph in which every component is a directed cycle.

In [15], Woodall (1972) gave the digraph version of Theorem $\AA$ as follows.

Theorem C (Woodall [15]) Let $D$ be a digraph of order $n \geq 2$. If $d_{D}^{+}(u)+d_{D}^{-}(v) \geq n$ for every two distinct vertices $u$ and $v$ with $(u, v) \notin A(D)$, then $D$ has a directed Hamilton cycle.

In fact, the following remark implies that this theorem is a generalization of Theorem $\mathrm{A}$.

Remark 1 For a given graph $G$, let $D_{G}$ be the digraph of order $|V(G)|$ obtained from $G$ by replacing each edge $u v$ in $G$ with two $\operatorname{arcs}(u, v)$ and $(v, u)$. It is easy to see that a graph $G$ satisfies the hypothesis (conclusion) of Theorem $\AA$ if and only if $D_{G}$ satisfies the hypothesis (conclusion) of Theorem $\mathrm{C}$, thus Theorem $\mathrm{C}$ implies Theorem $\mathrm{A}$,

In this paper, we show that the Woodall condition also implies the existence of the following directed 2-factor, which is our main result.

Theorem 1 Let $k$ be a positive integer, and let $D$ be a digraph of order $n$, where $n \geq 12 k+3$. If $d_{D}^{+}(u)+d_{D}^{-}(v) \geq n$ for every two distinct vertices $u$ and $v$ with $(u, v) \notin A(D)$, then $D$ has a directed 2-factor with exactly $k$ directed cycles of length at least 3.

By Remark 1, Theorem 1 implies the result of Theorem B for graphs with order $n \geq 12 k+3$, and it also clearly implies the result of Theorem $\mathbb{C}$ for graphs with order $n \geq 15$. Thus, in a sense, Theorem 1 is a common generalization of Theorems B and C (see Figure 1).

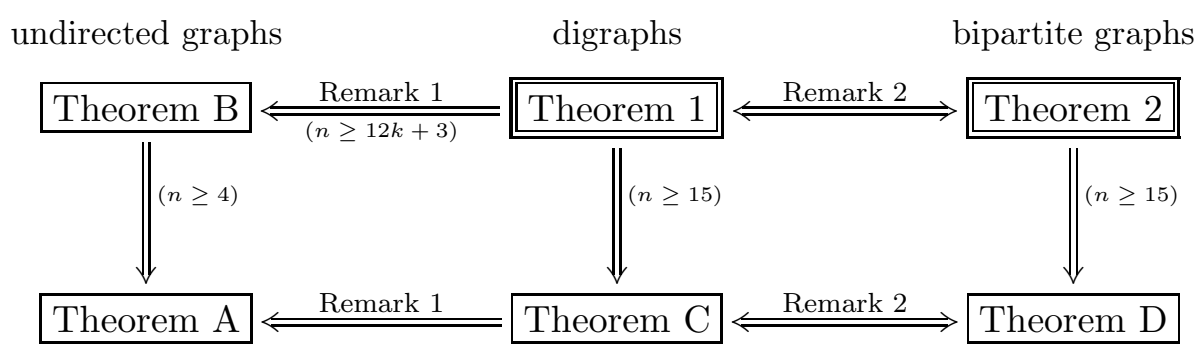

Figure 1: The relation between results in this paper

The degree condition in Theorem 1 is best possible in the following sense. Consider the complete bipartite graph $G=K_{(n-1) / 2,(n+1) / 2}$, and let $D_{G}$ be the digraph obtained from $G$ by the 
same procedure as in Remark 1. Then, $\min \left\{d_{D_{G}}^{+}(u)+d_{D_{G}}^{-}(v):(u, v) \notin A\left(D_{G}\right), u \neq v\right\}=n-1$ and clearly $D_{G}$ does not have a directed 2 -factor.

On the other hand, the order condition in Theorem 1 comes from our proof techniques. The complete bipartite graph $K_{2 k-1,2 k-1}$ shows that $n \geq 4 k-1$ is necessary for the existence of a 2 -factor with exactly $k$ cycles in simple undirected graphs, and hence it follows from the similar argument as above that $n \geq 4 k-1$ is also necessary for Theorem 1. Unfortunately, our proof of Theorem 1 requires the stronger condition $n \geq 12 k+3$; this arises from the condition $n \geq 12 k-9$ which is needed for the final contradiction in the proof of Theorem 4 (see Section 4).

In the next section, we further give a relationship between Theorem $\mathrm{C}$, Theorem 1 and the results on 2-factors containing perfect matchings in bipartite graphs (Theorem Dand Theorem 2) in Figure 1. The proof of Theorem 1 is presented in Sections 35 ,

\section{2-factors containing perfect matchings in bipartite graphs}

In previous section, in order to generalize Theorems $\mathrm{A}, \mathrm{B}$ and $\mathrm{C}$, we have considered the directed 2 -factors with exactly $k$ directed cycles of length at least 3 and have given Theorem 1 , It is also known that this problems have a connection with the 2-factor problems in bipartite graphs. In fact, Theorem [C is equivalent to the following theorem due to Las Vergnas (1972). Here, an edge subset $M$ of a graph $G$ is called a matching if no two edges in $M$ have a common end. In particular, a matching $M$ is said to be perfect if every vertex of $G$ is contained in some edge of $M$. An alternating cycle with respect to a matching $M$ is a cycle such that the edges belong to $M$ and not to $M$, alternatively. For a bipartite graph $G$ with partite sets $X$ and $Y$, we define $\sigma_{1,1}(G)=\min \left\{d_{G}(x)+d_{G}(y): x \in X, y \in Y, x y \notin E(G)\right\}$ if $G$ is not a complete bipartite graph; otherwise, $\sigma_{1,1}(G)=+\infty$.

Theorem D (Las Vergnas [8]) Let $G$ be a balanced bipartite graph of order $2 n \geq 4$ and $M$ be a perfect matching of $G$. If $\sigma_{1,1}(G) \geq n+2$, then $G$ has a Hamilton cycle containing every edge of $M$.

$D$

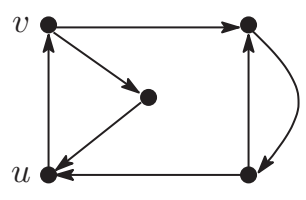

$G$

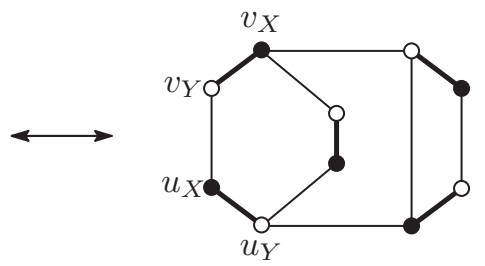

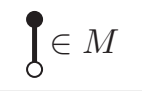

Figure 2: The relationship between digraphs and bipartite graphs with a perfect matching

Remark 2 (see also [16]) For a given digraph $D$, consider the following undirected graph $G$ : We split each vertex $v$ in $D$ into two vertices $v_{X}$ and $v_{Y}$ and replace each arc $(u, v)$ in 
$A(D)$ with a simple edge $u_{X} v_{Y}$, and we add the perfect matching $M=\left\{v_{X} v_{Y}: v \in V(D)\right\}$. Then, the resultant graph $G$ is a balanced bipartite graph of order $2|V(D)|$ with partite sets $\left\{v_{X}: v \in V(D)\right\}$ and $\left\{v_{Y}: v \in V(D)\right\}$ (see Figure 2). On the other hand, for a given balanced bipartite graph $G$ with partite sets $X, Y$ and a perfect matching $M$ in $G$, let $D$ be the digraph of order $|V(G)| / 2$ obtained from $G$ by replacing each edge $x y \in E(G) \backslash M(x \in X, y \in Y)$ with an arc from $x$ to $y$, and by contracting all edges of $M$ (see Figure 21). Note that, in this construction, the following hold:

- $(u, v) \in A(D)$ if and only if $u_{X} v_{Y} \in E(G)$ (in particular, $d_{D}^{+}(v)=d_{G}\left(v_{X}\right)-1$ and $\left.d_{D}^{-}(v)=d_{G}\left(v_{Y}\right)-1\right)$, and

- an alternating cycle of length $2 l(\geq 4)$ with respect to $M$ in $G$ corresponds to a directed cycle of length $l(\geq 2)$ in $D$.

Therefore, there is a one-to-one correspondence between the class of digraphs satisfying the Woodall condition and the class of bipartite graphs satisfying the Las Vergnas condition.

This also implies that Theorem 1 is equivalent to the following theorem (see also Figure 1). Related results can be found in [2] and a survey [7].

Theorem 2 Let $k$ be a positive integer, and let $G$ be a balanced bipartite graph of order $2 n$ and $M$ be a perfect matching of $G$, where $n \geq 12 k+3$. If $\sigma_{1,1}(G) \geq n+2$, then $G$ has a 2-factor with exactly $k$ cycles of length at least 6 containing every edge of $M$.

Proposition 3 Theorem 1 and Theorem 2 are equivalent.

Therefore, the Las Vergnas condition also implies the existence of a 2-factor with a prescribed number of cycles containing the specified perfect matching in bipartite graphs. In this sense, there is no difference between Hamilton cycles and 2-factors with $k(\geq 2)$ cycles.

\section{Proof of Theorem 1}

By Proposition 3, to prove Theorem 1, it suffices to show Theorem 2. Therefore, in this section, we introduce the steps of the proof of Theorem 2 and also give two theorems in order to prove it. Here, for a bipartite graph $G$ and a matching $M$ of $G$, a cycle $C$ of $G$ is called an $M$-cycle if $|E(C) \cap M|=\frac{|C|}{2}$, i.e., $C$ is an alternating cycle with respect to $M$. In particular, $C$ is called an $M$-Hamilton cycle if $C$ is a Hamilton cycle and an $M$-cycle, and a 2-factor of a graph $G$ is called an $M$-2-factor if every component is an $M$-cycle.

The proof of Theorem 2 involves two steps, summarized in the following two theorems.

Theorem 4 Let $k$ be a positive integer, and let $G$ be a balanced bipartite graph of order $2 n$ and $M$ be a perfect matching of $G$, where $n \geq 12 k-9$. If $\sigma_{1,1}(G) \geq n+2$, then $G$ contains $k$ disjoint $M$-cycles of length 6 or 8. 
Theorem 5 Let $k$ be a positive integer, and let $G$ be a balanced bipartite graph of order $2 n>6(k+1)$ and $M$ be a perfect matching of $G$. Suppose that $G$ contains $k+1$ disjoint $M$-cycles of length at least 6 . If $\sigma_{1,1}(G) \geq n+2$, then $G$ has an $M$-2-factor with exactly $k$ cycles of length at least 6.

Now we prove Theorem 1 assuming Theorems 4 and 5 .

Proof of Theorem 1. We first show that Theorem 2 is true. Let $k, n, G, M$ be the same as in Theorem 2. Since $n \geq 12 k+3=12(k+1)-9$ and $\sigma_{1,1}(G) \geq n+2$, it follows from Theorem 4 that $G$ contains $k+1$ disjoint $M$-cycles of length at least 6 . Then, since $n \geq 12 k+3>3(k+1)$, it follows from Theorem 5 that $G$ has an $M$-2-factor consisting of $k$ cycles of length at least 6 , that is, Theorem 2 is true. Then, by Proposition 3 , Theorem 1 is also true.

Therefore, it suffices to show that Theorems 4 and 5 hold. The proofs of Theorem 4 and 5 are presented in Sections 4 and 5, respectively. In Section 6, we mention a problem related to Theorem 4 .

Finally, we prepare terminology and notations, which will be used in the proofs. Let $G$ be a graph. We denote by $N_{G}(v)$ the neighborhood of a vertex $v$ in $G$. For $S \subseteq V(G)$, let $G[S]$ denote the subgraph induced by $S$ in $G$, and let $G-S=G[V(G) \backslash S]$. For $S, T \subseteq V(G)$ with $S \cap T=\emptyset$, $E_{G}(S, T)$ denotes the set of edges of $G$ between $S$ and $T$, and let $e_{G}(S, T)=\left|E_{G}(S, T)\right|$. We often identify a subgraph $H$ of $G$ with its vertex set $V(H)$ (e.g., we often use $E_{G}(F, H)$ instead of $E_{G}(V(F), V(H))$ for disjoint subgraphs $F$ and $H$ of $\left.G\right)$. We denote by $P[x, y]$ a path $P$ with ends $x$ and $y$ in $G$ and $|P|$ denotes the number of vertices in $P$. Next let $G$ be a bipartite graph, and $M$ be a matching of $G$. For a subgraph $H$ of $G$, let $M_{H}=M \cap E(H)$. A path $P=P[x, y]$ is called an $M$-path of $G$ if $P$ is an alternating path (i.e., a path such that the edges belong to $M$ and not to $M$, alternatively) joining $x$ and $y$ starting and ending with edges in $M$. In particular, $P$ is called an $M$-Hamilton path if $P$ is also a Hamilton path of $G$. If $X$ and $Y$ are partite sets of $G$, then for $A \subseteq X$ (resp., $B \subseteq Y$ ), we define $\bar{A}=\{y \in Y: x y \in M$ with $x \in A\}$ (resp., $\bar{B}=\{x \in X: x y \in M$ with $y \in B\}$ ).

\section{Proof of Theorem 4}

In this section, we give the proof of Theorem 4. In order to prove it, we use the following two lemmas (Lemmas 1 and 2).

Lemma 1 Let $G$ be a bipartite graph and $M$ be a matching of $G$, and let $C$ be an $M$-cycle and $P=P[x, y]$ be an $M$-path in $G-V(C)$. Assume that $e_{G}(\{x, y\}, C)>n$, where $n=\frac{|C|}{2}$. Then $G[V(P \cup C)]$ contains an $M$-cycle of length $|P|+2 i$ for each $i, 1 \leq i \leq n$.

Proof of Lemma 1, Let the vertices be labelled $u_{1} v_{1} \ldots u_{n} v_{n}$ in order round $C$, where $u_{1} v_{1}, \ldots$, $u_{n} v_{n}$ are the edges of $M_{C}$ and $v_{1}, \ldots, v_{n}$ are in the same partite set as $x$. If there is no $M$-cycle of length $|P|+2 i$, then $G$ contains at most one of the edges $x u_{j}, y v_{j+i-1}$ for each $j(i, j \in\{1, \ldots, n\})$, 
where the subscripts are interpreted modulo $n$, and so $e_{G}(\{x, y\}, C) \leq n$. This contradiction proves the result.

Lemma 2 Let $G$ be a balanced bipartite graph of order $2 n$ with partite sets $X$ and $Y$, and let $M$ be a perfect matching of $G$. If $d_{G}(x) \geq \frac{n+3}{2}$ for every vertex $x$ in $X$, then $G$ has an $M$-cycle of length 6 .

Proof of Lemma 2, Note that the hypothesis implies $n \geq \frac{n+3}{2}$, so that $n \geq 3$. Also, $\sum_{y \in Y} d_{G}(y)=|E(G)|=\sum_{x \in X} d_{G}(x) \geq n\left(\frac{n+3}{2}\right)$, which implies that there exists a vertex $y$ in $Y$ such that $d_{G}(y) \geq \frac{n+3}{2}$.

Suppose that $G$ contains no $M$-cycle of length 6 . Let $x$ be a vertex in $X$ such that $x y \in M$, and let $x^{\prime} y^{\prime} \in M \backslash\{x y\}$ such that $x y^{\prime} \in E(G)$. Then $N_{G}(y) \cap\left(\overline{N_{G}\left(x^{\prime}\right)} \backslash\left\{x, x^{\prime}\right\}\right)=\emptyset$ since otherwise, $G$ contains an $M$-cycle of length 6 , a contradiction. Note that $\left|\overline{N_{G}\left(x^{\prime}\right)}\right|=\left|N_{G}\left(x^{\prime}\right)\right|=$ $d_{G}\left(x^{\prime}\right) \geq \frac{n+3}{2}$, ans so

$$
d_{G}(y) \leq|X|-\left|\overline{N_{G}\left(x^{\prime}\right)}\right|+2 \leq n-\frac{n+3}{2}+2=\frac{n+1}{2}<\frac{n+3}{2},
$$

a contradiction.

Now we are ready to prove Theorem 4 ,

Proof of Theorem 4. If $n=3$, then by the degree condition, we can easily check that $G$ is a complete bipartite graph, and hence the assertion clearly holds. Thus, we may assume that $n \geq 4$, i.e., $n \geq \max \{4,12 k-9\}$.

We suppose that

$G$ contains $t$ disjoint $M$-cycles of length 6 or 8 with $0 \leq t \leq k-1$, but $G$ does not contain $t+1$ disjoint $M$-cycles of length 6 or 8 .

Let $C_{1}, \ldots, C_{t}$ be $t$ disjoint $M$-cycles of length 6 or 8 , and choose $C_{1}, \ldots, C_{t}$ so that $\sum_{i=1}^{t}\left|C_{i}\right|$ is as small as possible. Without loss of generality, we may assume that $C_{1}, \ldots, C_{t_{1}}$ are cycles of length 6 and $C_{t_{1}+1}, \ldots, C_{t}$ are cycles of length 8 for some $t_{1}$ with $0 \leq t_{1} \leq t$. Let $H=G-\bigcup_{i=1}^{t} V\left(C_{i}\right)$.

Now let $\mathcal{P}$ be a set of mutually disjoint $M$-paths of order 4 in $H$, and we define

$$
\mathcal{P}^{*}=\left\{P[x, y] \in \mathcal{P}: d_{G}(x)+d_{G}(y) \geq \sigma_{1,1}(G)\right\} .
$$

We choose $\mathcal{P}$ so that

(P1) $\left|\mathcal{P}^{*}\right|$ is as large as possible, and

(P2) $|\mathcal{P}|$ is as large as possible, subject to (P1).

Claim 4.1 $|\mathcal{P}| \geq k+1$. 
Proof. Note that $|H| \geq 2 n-8 t \geq 2 n-8(k-1)=2 n-8 k+8$. Suppose that $|\mathcal{P}| \leq k$, and let $H^{\prime}=H-\bigcup_{P \in \mathcal{P}} V(P)$. Then $\left|H^{\prime}\right| \geq|H|-4 k \geq 2 n-12 k+8 \geq 2 \max \{4,12 k-9\}-12 k+8 \geq 4$, and so there are two distinct edges $x_{1} y_{1}$ and $x_{2} y_{2}$ in $M_{H^{\prime}}$ (note that $M_{H^{\prime}}$ is a perfect matching of $H^{\prime}$ ), where $x_{1}$ and $x_{2}$ belong to the same partite set of $G$. Then $d_{H^{\prime}}\left(x_{h}\right)=d_{H^{\prime}}\left(y_{h}\right)=1$ for $h \in\{1,2\}$, as otherwise $H^{\prime}$ has an $M$-path of order 4, which contradicts (P2). In particular, $x_{1} y_{2}, x_{2} y_{1} \notin$ $E(G)$. Also, $e_{G}\left(\left\{x_{h}, y_{h}\right\}, C_{i}\right) \leq\left|C_{i}\right|=6$ for $h \in\{1,2\}$ and $1 \leq i \leq t_{1} ; e_{G}\left(\left\{x_{h}, y_{h}\right\}, C_{i}\right) \leq 4<6$ for $h \in\{1,2\}$ and $t_{1}+1 \leq i \leq t$, since otherwise $G\left[\left\{x_{h}, y_{h}\right\} \cup V\left(C_{i}\right)\right]$ contains an $M$-cycle $C_{i}^{\prime}$ of length 6 by Lemma 1, and replacing the cycle $C_{i}$ by this cycle $C_{i}^{\prime}$ would violate the minimality of $\sum_{i=1}^{t}\left|C_{i}\right|$; and $e_{G}\left(\left\{x_{h}, y_{h}\right\}, P\right) \leq 3$ for $h \in\{1,2\}$ and each $P \in \mathcal{P}$, since otherwise, it is easy to see that $G\left[\left\{x_{h}, y_{h}\right\} \cup V(P)\right]$ has an $M$-cycle of length 6 , which contradicts (4.1). Since $x_{1} y_{2}, x_{2} y_{1} \notin E(G)$, it follows that

$$
\begin{aligned}
2 n+4 & \leq 2 \sigma_{1,1}(G) \\
& \leq\left(d_{G}\left(x_{1}\right)+d_{G}\left(y_{2}\right)\right)+\left(d_{G}\left(x_{2}\right)+d_{G}\left(y_{1}\right)\right) \\
& =\left(d_{G}\left(x_{1}\right)+d_{G}\left(y_{1}\right)\right)+\left(d_{G}\left(x_{2}\right)+d_{G}\left(y_{2}\right)\right) \\
& \leq 2(2+6 t+3 k) \\
& \leq 2(2+6(k-1)+3 k)=18 k-8,
\end{aligned}
$$

which implies $n \leq 9 k-6<\max \{4,12 k-9\}$, a contradiction.

Claim $4.2\left|\mathcal{P}^{*}\right| \geq|\mathcal{P}|-1$.

Proof. Suppose that $\left|\mathcal{P}^{*}\right| \leq|\mathcal{P}|-2$, and let $P_{1}\left[x_{1}, y_{1}\right], P_{2}\left[x_{2}, y_{2}\right] \in \mathcal{P} \backslash \mathcal{P}^{*}$ with $P_{1} \neq P_{2}$. Since $P_{1}, P_{2} \notin \mathcal{P}^{*}$, we have $x_{1} y_{1}, x_{2} y_{2} \in E(G)$. We may assume that $x_{1}$ and $x_{2}$ belong to the same partite set of $G$. If $E_{G}\left(\left\{x_{1}, y_{1}\right\},\left\{x_{2}, y_{2}\right\}\right)=\emptyset$, then

$$
\sum_{h \in\{1,2\}}\left(d_{G}\left(x_{h}\right)+d_{G}\left(y_{h}\right)\right)=\left(d_{G}\left(x_{1}\right)+d_{G}\left(y_{2}\right)\right)+\left(d_{G}\left(x_{2}\right)+d_{G}\left(y_{1}\right)\right) \geq 2 \sigma_{1,1}(G),
$$

and this implies that $d_{G}\left(x_{1}\right)+d_{G}\left(y_{1}\right) \geq \sigma_{1,1}(G)$ or $d_{G}\left(x_{2}\right)+d_{G}\left(y_{2}\right) \geq \sigma_{1,1}(G)$, which contradicts the assumption that $P_{1}, P_{2} \notin \mathcal{P}^{*}$. Thus $E_{G}\left(\left\{x_{1}, y_{1}\right\},\left\{x_{2}, y_{2}\right\}\right) \neq \emptyset$. We will assume that $x_{1} y_{2} \in$ $E(G)$.

Write $P_{1}=x_{1} y_{1}^{\prime} x_{1}^{\prime} y_{1}$ and $P_{2}=x_{2} y_{2}^{\prime} x_{2}^{\prime} y_{2}$. Note that $x_{h} y_{h}^{\prime}, x_{h}^{\prime} y_{h} \in M$ for $h \in\{1,2\}$. Consider the $M$-path $Q=y_{1}^{\prime} x_{1} y_{2} x_{2}^{\prime}$. If $y_{1}^{\prime} x_{2}^{\prime} \notin E(G)$, then $Q\left[y_{1}^{\prime}, x_{2}^{\prime}\right]$ is an $M$-path of order 4 such that $d_{G}\left(y_{1}^{\prime}\right)+d_{G}\left(x_{2}^{\prime}\right) \geq \sigma_{1,1}(G)$, and hence for $\mathcal{Q}=\left(\mathcal{P} \backslash\left\{P_{1}, P_{2}\right\}\right) \cup\{Q\}$, we have $\left|\mathcal{Q}^{*}\right|>\left|\mathcal{P}^{*}\right|$ because $P_{1}, P_{2} \notin \mathcal{P}^{*}$, which contradicts $(\mathrm{P} 1)$. Thus $y_{1}^{\prime} x_{2}^{\prime} \in E(G)$. Since $x_{1} y_{1}, x_{1} y_{2}, y_{1}^{\prime} x_{2}^{\prime} \in E(G)$, it follows that $y_{1}^{\prime} x_{2}, x_{1}^{\prime} y_{2} \notin E(G)$; otherwise, $x_{2} y_{2}^{\prime} x_{2}^{\prime} y_{2} x_{1} y_{1}^{\prime} x_{2}$ or $x_{1}^{\prime} y_{1} x_{1} y_{1}^{\prime} x_{2}^{\prime} y_{2} x_{1}^{\prime}$ is an $M$-cycle of length 6 , which contradicts (4.1). Therefore,

$$
\begin{aligned}
& \left(d_{G}\left(x_{1}^{\prime}\right)+d_{G}\left(y_{1}^{\prime}\right)\right)+\left(d_{G}\left(x_{2}\right)+d_{G}\left(y_{2}\right)\right) \\
= & \left(d_{G}\left(x_{1}^{\prime}\right)+d_{G}\left(y_{2}\right)\right)+\left(d_{G}\left(y_{1}^{\prime}\right)+d_{G}\left(x_{2}\right)\right) \geq 2 \sigma_{1,1}(G),
\end{aligned}
$$

and this implies that $d_{G}\left(x_{1}^{\prime}\right)+d_{G}\left(y_{1}^{\prime}\right) \geq \sigma_{1,1}(G)$ or $d_{G}\left(x_{2}\right)+d_{G}\left(y_{2}\right) \geq \sigma_{1,1}(G)$. Since $P_{2} \in \mathcal{P} \backslash \mathcal{P}^{*}$, we have $d_{G}\left(x_{1}^{\prime}\right)+d_{G}\left(y_{1}^{\prime}\right) \geq \sigma_{1,1}(G)$. 
Now consider the $M$-path $R_{1}=x_{1}^{\prime} y_{1} x_{1} y_{1}^{\prime}$, and let $\mathcal{R}=\left(\mathcal{P} \backslash\left\{P_{1}\right\}\right) \cup\left\{R_{1}\right\}$. Since $P_{1}\left[x_{1}, y_{1}\right] \in$ $\mathcal{P} \backslash \mathcal{P}^{*}$ and $R_{1}\left[x_{1}^{\prime}, y_{1}^{\prime}\right] \in \mathcal{R}^{*}$, we have $\left|\mathcal{R}^{*}\right|>\left|\mathcal{P}^{*}\right|$, which contradicts (P1) again.

Claim 4.3 There exist at least two distinct paths $P[x, y]$ in $\mathcal{P}$ such that $e_{G}\left(\{x, y\}, C_{i}\right) \leq 4$ for $1 \leq i \leq t_{1}$.

Proof. Suppose not. Then, for every path $P[x, y]$ in $\mathcal{P}$, except at most one, there exists a cycle $C_{i}$ with $1 \leq i \leq t_{1}$ such that $e_{G}\left(\{x, y\}, C_{i}\right) \geq 5$. Since $|\mathcal{P}| \geq k+1$ by Claim 4.1, and since $t_{1} \leq k-$ 1 , it follows from the Pigeonhole Principle that there exist two distinct paths $P[x, y], P^{\prime}\left[x^{\prime}, y^{\prime}\right]$ in $\mathcal{P}$ and a cycle $C_{i}$ with $1 \leq i \leq t_{1}$ such that $e_{G}\left(\{x, y\}, C_{i}\right) \geq 5$ and $e_{G}\left(\left\{x^{\prime}, y^{\prime}\right\}, C_{i}\right) \geq 5$. Hence we can take two distinct edges $u v, u^{\prime} v^{\prime}$ in $M_{C_{i}}$ such that $e_{G}(\{x, y\},\{u, v\})=e_{G}\left(\left\{x^{\prime}, y^{\prime}\right\},\left\{u^{\prime}, v^{\prime}\right\}\right)=$ 2 , and then it is easy to check that $G\left[V\left(P \cup P^{\prime}\right) \cup\left\{u, v, u^{\prime}, v^{\prime}\right\}\right]$ contains two disjoint $M$-cycles of length 6, which contradicts (4.1).

By Claims 4.2 and 4.3 , there exists an $M$-path $P_{0}\left[x_{0}, y_{0}\right]$ in $\mathcal{P}$ such that $P_{0} \in \mathcal{P}^{*}$ and $e_{G}\left(\left\{x_{0}, y_{0}\right\}, C_{i}\right) \leq 4$ for $1 \leq i \leq t_{1}$. Note that $e_{G}\left(\left\{x_{0}, y_{0}\right\}, C_{i}\right) \leq \frac{\left|C_{i}\right|}{2}=4$ for $t_{1}+1 \leq i \leq t$ as well, as otherwise $G\left[V\left(P_{0} \cup C_{i}\right)\right]$ contains an $M$-cycle of length $\left|P_{0}\right|+2=6$ by Lemma 1, and replacing $C_{i}$ by this new cycle would violate the minimality of $\sum_{i=1}^{t}\left|C_{i}\right|$. Let $H^{\prime}=H-V\left(P_{0}\right)$. Then

$$
\begin{aligned}
e_{G}\left(\left\{x_{0}, y_{0}\right\}, H^{\prime}\right) & \geq \sigma_{1,1}(G)-\sum_{i=1}^{t} e_{G}\left(\left\{x_{0}, y_{0}\right\}, C_{i}\right)-d_{G\left[V\left(P_{0}\right)\right]}\left(x_{0}\right)-d_{G\left[V\left(P_{0}\right)\right]}\left(y_{0}\right) \\
& \geq(n+2)-4 t-2-2 \\
& =n-4 t-2 .
\end{aligned}
$$

We let

$$
\begin{array}{ll}
Y_{x_{0}}=N_{H}\left(x_{0}\right) \cap V\left(H^{\prime}\right), & X_{x_{0}}=\overline{Y_{x_{0}}}, \\
X_{y_{0}}=N_{H}\left(y_{0}\right) \cap V\left(H^{\prime}\right), & Y_{y_{0}}=\overline{X_{y_{0}}} .
\end{array}
$$

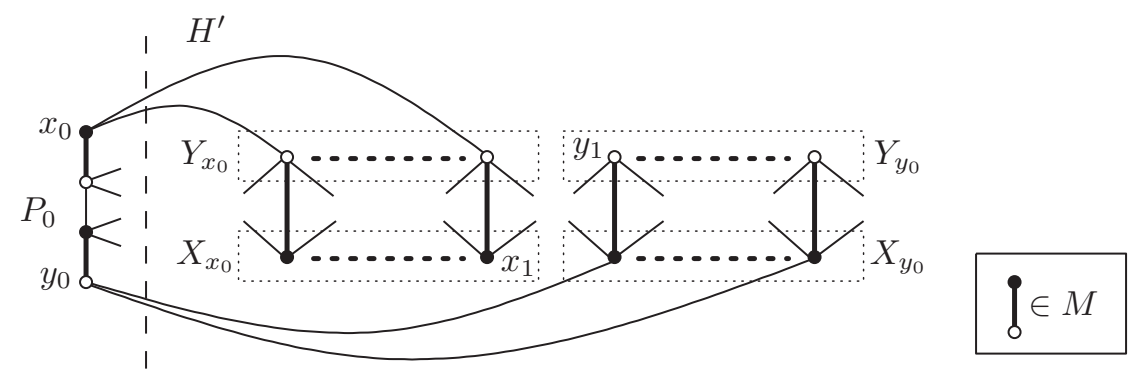

Figure 3: Vertex subsets $X_{x_{0}}, Y_{x_{0}}, X_{y_{0}}$ and $Y_{y_{0}}$

Since $H$ does not contain an $M$-cycle of length 6 by (4.1), we have $X_{x_{0}} \cap X_{y_{0}}=Y_{x_{0}} \cap Y_{y_{0}}=\emptyset$, and hence

$$
X_{x_{0}}, Y_{x_{0}}, X_{y_{0}} \text { and } Y_{y_{0}} \text { are pairwise disjoint. }
$$


Note also that

$$
e_{G}\left(x_{0}, H^{\prime}\right)=\left|X_{x_{0}}\right|=\left|Y_{x_{0}}\right| \text { and } e_{G}\left(y_{0}, H^{\prime}\right)=\left|X_{y_{0}}\right|=\left|Y_{y_{0}}\right|
$$

Let $G_{x_{0}}=G\left[X_{x_{0}} \cup Y_{x_{0}}\right]$ and $G_{y_{0}}=G\left[X_{y_{0}} \cup Y_{y_{0}}\right]$. Then by (4.3)-(4.5), it follows that $G_{x_{0}}$ and $G_{y_{0}}$ are disjoint balanced bipartite graphs with a perfect matching whose edges belong to $M$. We further define

$$
\begin{aligned}
& n_{1}=\sum_{i=1}^{t} \frac{\left|C_{i}\right|}{2}, \quad n_{2}=\frac{\left|G_{x_{0}}\right|}{2}=e_{G}\left(x_{0}, H^{\prime}\right), \quad n_{3}=\frac{\left|G_{y_{0}}\right|}{2}=e_{G}\left(y_{0}, H^{\prime}\right), \\
& n_{4}=2+\frac{\left|H^{\prime}\right|}{2}-\left(\frac{\left|G_{x_{0}}\right|}{2}+\frac{\left|G_{y_{0}}\right|}{2}\right) .
\end{aligned}
$$

Claim 4.4 If $n_{2}>0$ (resp., $n_{3}>0$ ), then there exists a vertex $x_{1}$ in $X_{x_{0}}$ (resp., a vertex $y_{1}$ in $\left.Y_{y_{0}}\right)$ such that $d_{G_{x_{0}}}\left(x_{1}\right) \leq \frac{n_{2}+2}{2}\left(\right.$ resp., $\left.d_{G_{y_{0}}}\left(y_{1}\right) \leq \frac{n_{3}+2}{2}\right)$.

Proof. Suppose that $n_{2}>0$ and that $d_{G_{x_{0}}}(x) \geq \frac{n_{2}+3}{2}$ for every vertex $x$ in $X_{x_{0}}$. Since $M_{G_{x_{0}}}=$ $M \cap E\left(G_{x_{0}}\right)$ is a perfect matching of $G_{x_{0}}$ and $d_{G_{x_{0}}}(x) \geq \frac{n_{2}+3}{2}$ for $x \in X_{x_{0}}$, it follows from Lemma 2 that $G_{x_{0}}$ has an $M$-cycle of length 6, which contradicts (4.1).

By symmetry, if $n_{3}>0$, then there exists a vertex $y_{1}$ in $Y_{y_{0}}$ such that $d_{G_{y_{0}}}\left(y_{1}\right) \leq \frac{n_{3}+2}{2}$.

By (4.2) and (4.6), and since $t \leq k-1$ and $n \geq 12 k-9$, we have

$$
n_{2}+n_{3}=e_{G}\left(\left\{x_{0}, y_{0}\right\}, H^{\prime}\right) \geq n-4 t-2 \geq 8 k-7>0 .
$$

In particular, without loss of generality, we may assume that $n_{2}>0$. Then by Claim 4.4 , there exists a vertex $x_{1}$ in $X_{x_{0}}$ such that $d_{G_{x_{0}}}\left(x_{1}\right) \leq \frac{n_{2}+2}{2}$. If $n_{3}>0$, then let $y_{1}$ be also the vertex as in Claim 4.4, otherwise, let $y_{1}=y_{0}$. Note that

$$
\left.N_{G}\left(x_{1}\right) \cap\left(\left\{y_{0}\right\}\right) \cup Y_{y_{0}}\right)=\emptyset
$$

since otherwise, $H$ has an $M$-cycle of length 6 or 8 , which contradicts (4.1).

Claim 4.5 We have $d_{G}\left(x_{1}\right) \leq n_{1}+\frac{n_{2}}{2}+n_{4}$ and $d_{G}\left(y_{1}\right) \leq n_{1}+\frac{n_{3}}{2}+n_{4}$.

Proof. We first show that $d_{G}\left(x_{1}\right) \leq n_{1}+\frac{n_{2}}{2}+n_{4}$. Note that $e_{G}\left(x_{1}, P_{0}\right) \leq 1$ since $x_{1} y_{1} \notin E(G)$ by (4.8). Combining this with (4.3)-(4.6), (4.8) and the definition of $x_{1}$, we get

$$
\begin{aligned}
d_{G}\left(x_{1}\right) & =\sum_{i=1}^{t} e_{G}\left(x_{1}, C_{i}\right)+e_{G}\left(x_{1}, P_{0}\right)+d_{H^{\prime}}\left(x_{1}\right) \\
& \leq n_{1}+1+\left(d_{G_{x_{0}}}\left(x_{1}\right)+\left|N_{H^{\prime}}\left(x_{1}\right) \cap Y_{y_{0}}\right|+\left(\frac{\left|H^{\prime}\right|}{2}-\left|Y_{x_{0}}\right|-\left|Y_{y_{0}}\right|\right)\right) \\
& \leq n_{1}+1+\left(\frac{n_{2}+2}{2}+0+\left(\frac{\left|H^{\prime}\right|}{2}-\frac{\left|G_{x_{0}}\right|}{2}-\frac{\left|G_{y_{0}}\right|}{2}\right)\right) \\
& =n_{1}+\frac{n_{2}}{2}+\left(2+\frac{\left|H^{\prime}\right|}{2}-\frac{\left|G_{x_{0}}\right|}{2}-\frac{\left|G_{y_{0}}\right|}{2}\right)=n_{1}+\frac{n_{2}}{2}+n_{4} .
\end{aligned}
$$


We next show that $d_{G}\left(y_{1}\right) \leq n_{1}+\frac{n_{3}}{2}+n_{4}$. If $n_{3}>0$, then this holds by the same argument. Thus we may assume $n_{3}=0$. Recall that, in this case, $y_{1}=y_{0}$, and note that by (4.6), $e_{G}\left(y_{1}, H^{\prime}\right)=n_{3}=0$ and $n_{4} \geq 2$. Hence,

$$
d_{G}\left(y_{1}\right)=\sum_{i=1}^{t} e_{G}\left(y_{1}, C_{i}\right)+d_{G\left[V\left(P_{0}\right)\right]}\left(y_{1}\right) \leq n_{1}+2 \leq n_{1}+\frac{n_{3}}{2}+n_{4} .
$$

Since $x_{1} y_{1} \notin E(G)$ by (4.8), it follows from Claim 4.5) and the hypothesis of Theorem 4 that

$$
n+2 \leq \sigma_{1,1}(G) \leq d_{G}\left(x_{1}\right)+d_{G}\left(y_{1}\right) \leq 2 n_{1}+\frac{n_{2}+n_{3}}{2}+2 n_{4} .
$$

This inequality implies that $2(n+2) \leq 4 n_{1}+n_{2}+n_{3}+4 n_{4}$. Since $n=n_{1}+n_{2}+n_{3}+n_{4}$ by (4.6) and the fact that $|H|=\left|H^{\prime}\right|+\left|P_{0}\right|=\left|H^{\prime}\right|+4$, it follows that $n \leq 3\left(n_{1}+n_{4}\right)-4$. By (4.7), we obtain $n_{1}+n_{4}=n-\left(n_{2}+n_{3}\right) \leq 4 t+2$. Thus we have

$$
n \leq 3(4 t+2)-4=12 t+2 \leq 12 k-10<12 k-9,
$$

a contradiction.

This completes the proof of Theorem 4

\section{Proof of Theorem 5}

We first prepare terminology and notation. Let $G$ be a graph. We write a cycle (or a path) $C$ with a given orientation as $\vec{C}$. Let $\vec{C}$ be an oriented cycle (or path). For $x \in V(C)$, we denote the successor and the predecessor of $x$ on $\vec{C}$ by $x^{+}$and $x^{-}$. For $X \subseteq V(C)$, let $X^{-}=\left\{x^{-}: x \in V(C)\right\}$. For $x, y \in V(C)$, we denote by $x \vec{C} y$ the path with ends $x$ and $y$ on $\vec{C}$. The reverse sequence of $x \vec{C} y$ is denoted by $y \overleftarrow{C} x$. In the rest of this paper, we assume that every cycle has a fixed orientation.

In order to prove Theorem 5, we give three lemmas as follows (Lemmas 3 5). (We omit the proof of Lemma 3 since it is easy and it is similar to the proof of Lemma 1 in Section 4).

Lemma 3 Let $G$ be a bipartite graph and $M$ be a matching of $G, C$ be an $M$-cycle or an $M$-path, and $x$ and $y$ be two vertices of $G-V(C)$ which belong to different partite sets of $G$.

(1) If $C$ is a cycle and $e_{G}(\{x, y\}, C) \geq \frac{|C|}{2}+1$, then there exists an edge uv in $E(C) \backslash M$ such that $e_{G}(\{x, y\},\{u, v\})=2$.

(2) If $C$ is a path and $e_{G}(\{x, y\}, C) \geq \frac{|C|}{2}+2$, then there exists an edge uv in $E(C) \backslash M$ such that $e_{G}(\{x, y\},\{u, v\})=2$.

Before stating Lemmas 4 and 5, we further prepare the following terminology. Let $G$ be a bipartite graph and $M$ be a matching of $G$. For an $M$-cycle $C$ (resp., an $M$-path $C$ ) and an $M$-path $Q[x, y]$ such that $V(C) \cap V(Q)=\emptyset, Q$ is insertible in $C$ if there exists an edge $u v$ in 
$E(C) \backslash M$ such that $e_{G}(\{x, y\},\{u, v\})=2$. We call the edge $u v$ an insertion edge of $Q$. Note that by Lemma 3, if $C$ is a cycle and $e_{G}(\{x, y\}, C) \geq \frac{|C|}{2}+1$, then $Q$ is insertible in $C$; if $C$ is a path and $e_{G}(\{x, y\}, C) \geq \frac{|C|}{2}+2$, then $Q$ is insertible in $C$. Note also that if $C$ is a cycle and $Q$ is insertible in $C$, then $G[V(C \cup Q)]$ has an $M_{C \cup Q}$-Hamilton cycle; if $C$ is a path and $Q$ is insertible in $C$, then $G[V(C \cup Q)]$ has an $M_{C \cup Q}$-Hamilton path such that the ends of the path are the same ends as $C$. An $M$-path $P$ is maximal if there exists no $M$-path $Q$ such that $|P|<|Q|$ and $V(P) \subseteq V(Q)$.

Lemma 4 Let $G$ be a balanced bipartite graph of order $2 n, M$ be a perfect matching of $G$, and $D_{1}, \ldots, D_{s}$ be $s$ disjoint $M$-cycles of length at least 6 in $G$. Let $H=G-\bigcup_{i=1}^{s} V\left(D_{i}\right)$, and $\overrightarrow{P_{0}}[x, y]$ be a maximal $M$-path of order at least 2 in $H$. If $\sigma_{1,1}(G) \geq n+2$, then one of the following (ii)-(iii) holds:

(i) either $\left|P_{0}\right|=2$ or $G\left[V\left(P_{0}\right)\right]$ has an $M_{P_{0}}$-Hamilton cycle,

(ii) for some cycle $D_{i}$ with $1 \leq i \leq s, G\left[V\left(P_{0} \cup D_{i}\right)\right]$ has an $M_{P_{0} \cup D_{i}}$-2-factor with exactly two cycles $D_{0}$ and $D_{i}^{\prime}$ of length at least 6 such that $V\left(D_{i}\right) \subsetneq V\left(D_{i}^{\prime}\right)$,

(iii) $e_{G}(\{x, y\}, G-H) \geq|G-H| / 2+1$.

Proof of Lemma 4. Suppose that each of (ii) and (iii) does not hold; we show that (iii) holds. Since (ii) does not hold, we have $x y \notin E(G)$, and hence $d_{G}(x)+d_{G}(y) \geq n+2$. Since $P_{0}$ is maximal, we obtain $e_{G}\left(\{x, y\}, H-P_{0}\right)=0$. Therefore, it suffices to show that $d_{G\left[V\left(P_{0}\right)\right]}(x)+$ $d_{G\left[V\left(P_{0}\right)\right]}(y) \leq \frac{\left|P_{0}\right|}{2}+1$, since this will imply that

$$
e_{G}(\{x, y\}, G-H) \geq(n+2)-\left(\frac{\left|P_{0}\right|}{2}+1\right) \geq \frac{|G-H|}{2}+1 .
$$

Suppose that $d_{G\left[V\left(P_{0}\right)\right]}(x)+d_{G\left[V\left(P_{0}\right)\right]}(y) \geq \frac{\left|P_{0}\right|}{2}+2$. Let $v$ be the first vertex along $\vec{P}_{0}$ that is in $N_{G}(y)$, and let $u$ be the last vertex along $\overrightarrow{P_{0}}$ that is in $N_{G}(x)$. Since all vertices of $N_{G\left[V\left(P_{0}\right)\right]}(x)^{-}$ and $N_{G\left[V\left(P_{0}\right)\right]}(y)$ are in the same partite set, it follows that there are at most $\left|P_{0}\right| / 2$ vertices in the union of these two sets and hence at least two vertices in their intersection. It follows from this and the fact that $x y \notin E(G)$ that the vertices $x, v, u, y$ are distinct and occur in this order along $\overrightarrow{P_{0}}$, and $u \neq v^{+}$. Let $\overrightarrow{D_{0}}$ be the $M$-cycle $x \overrightarrow{P_{0}} u x$ and let $\overrightarrow{Q_{0}}[w, z]$ be the $M$-path $u^{+} \overrightarrow{P_{0}} y$ (see the left figure of Figure 4). Then the following hold:

(A) $V\left(D_{0}\right) \cap V\left(Q_{0}\right)=\emptyset$ and $V\left(D_{0}\right) \cup V\left(Q_{0}\right)=V\left(P_{0}\right)$;

(B) there exist two vertices $u, v \in V\left(D_{0}\right)$ such that $u v \notin M$ and $w u, z v \in E(G)$;

(C) $\left|D_{0}\right| \geq 6$.

In the rest of this proof, we use only $(\mathrm{A})-(\mathrm{C})$. We make no further use of the orientation of $\overrightarrow{P_{0}}$; the superscripts ${ }^{+}$and ${ }^{-}$will refer to the orientations of $\overrightarrow{D_{0}}$ and $\overrightarrow{Q_{0}}$. We assume $\overrightarrow{D_{0}}$ is oriented so that $u u^{+} \in E\left(D_{0}\right) \backslash M$. Note that $u^{+} \neq v$ since (ii) does not hold. Choose $D_{0}, Q_{0}$ and $u, v$ so that $(\mathrm{A})-(\mathrm{C})$ hold and 


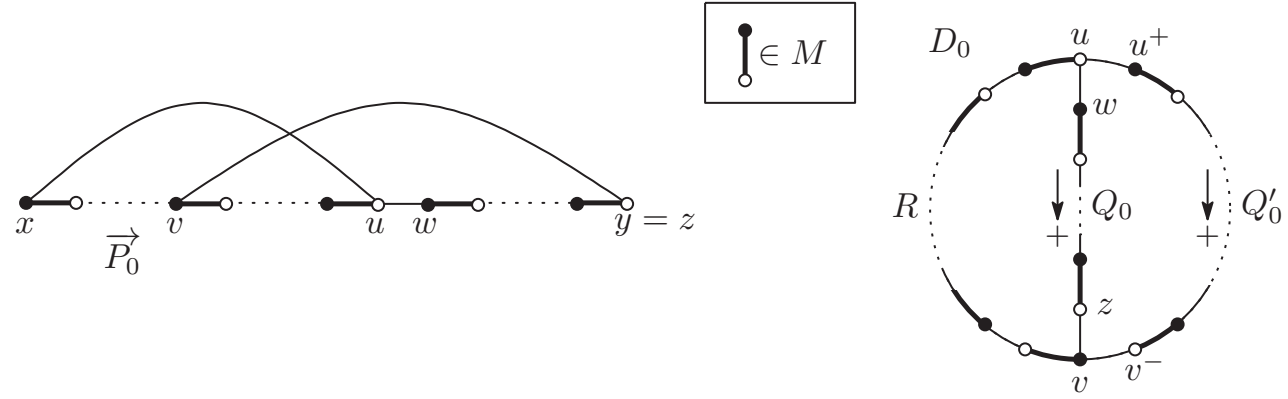

Figure 4: The $M$-cycle $D_{0}$ and the $M$-path $Q_{0}$

(D1) $\left|D_{0}\right|$ is as large as possible, and

(D2) $\left|u \overrightarrow{D_{0}} v\right|$ is as small as possible, subject to (D1).

Let $D_{0}^{\prime}=v \overrightarrow{D_{0}} u w \overrightarrow{Q_{0}} z v$ and $Q_{0}^{\prime}=u^{+} \overrightarrow{D_{0}} v^{-}$, and let $R=v \overrightarrow{D_{0}} u$ (note that $D_{0}^{\prime}=Q_{0} \cup R$ ). See the right figure of Figure 4.

Suppose that $N_{G}(w) \cap V\left(Q_{0}^{\prime}\right) \neq \emptyset$, say $b \in N_{G}(w) \cap V\left(Q_{0}^{\prime}\right)$. Then $\left|b \overrightarrow{D_{0}} v\right|<\left|u \overrightarrow{D_{0}} v\right|$ and $b v \notin M$. Replacing the pair of vertices $(u, v)$ with $(b, v)$, this contradicts (D2). Thus $N_{G}(w) \cap$ $V\left(Q_{0}^{\prime}\right)=\emptyset$. Similarly, we have $N_{G}(z) \cap V\left(Q_{0}^{\prime}\right)=\emptyset$.

Suppose next that $N_{G}\left(u^{+}\right) \cap V\left(Q_{0}\right) \neq \emptyset$, say $b \in N_{G}\left(u^{+}\right) \cap V\left(Q_{0}\right)$. Consider the $M$-cycle $D=w \overrightarrow{Q_{0}} b u^{+} \overrightarrow{D_{0}} u w$. If $b=z$, then (ii) holds, contradicting our assumption. Thus $b \neq z$, and we further consider the $M$-path $Q=b^{+} \overrightarrow{Q_{0}} z$. Then $V(D) \cap V(Q)=\emptyset$ and $V(D) \cup V(Q)=V\left(P_{0}\right)$. Furthermore, $|D|>\left|D_{0}\right| \geq 6$ and $b^{+} b, z v$ are two independent edges with $b, v \in V(D)$ and $b v \notin M$. This contradicts (D1). Thus $N_{G}\left(u^{+}\right) \cap V\left(Q_{0}\right)=\emptyset$. By the similar way, we see that $N_{G}\left(v^{-}\right) \cap V\left(Q_{0}\right)=\emptyset$.

By the above arguments, we can get

$$
e_{G}\left(\{w, z\}, Q_{0}^{\prime}\right)=e_{G}\left(\left\{u^{+}, v^{-}\right\}, Q_{0}\right)=0 .
$$

Moreover, we clearly have

$$
d_{G\left[V\left(Q_{0}\right)\right]}(w)+d_{G\left[V\left(Q_{0}\right)\right]}(z) \leq\left|Q_{0}\right| \text { and } d_{G\left[V\left(Q_{0}^{\prime}\right)\right]}\left(u^{+}\right)+d_{G\left[V\left(Q_{0}^{\prime}\right)\right]}\left(v^{-}\right) \leq\left|Q_{0}^{\prime}\right| .
$$

On the other hand, since (i) does not hold, it follows that $Q_{0}$ is not insertible in $D_{0}$. This in particular implies that $Q_{0}$ is not insertible in the $M$-path $R$. Similarly, $Q_{0}^{\prime}$ is not insertible in $D_{0}^{\prime}$, and hence $Q_{0}^{\prime}$ is not also insertible in the $M$-path $R$. Therefore, by Lemma 3(2), we get

$$
e_{G}(\{w, z\}, R) \leq \frac{|R|}{2}+1 \text { and } e_{G}\left(\left\{u^{+}, v^{-}\right\}, R\right) \leq \frac{|R|}{2}+1 .
$$

Recall that by (C), $\left|D_{0}\right| \geq 6$. Since (iii) does not hold, it follows that $Q_{0}$ is not insertible in each $D_{i}(1 \leq i \leq s)$. Moreover, since $u v \notin M$ by $(\mathrm{B})$, we have $|R| \geq 4$, that is, $\left|D_{0}^{\prime}\right|=$ $\left|Q_{0}\right|+|R| \geq 6$. This implies that $Q_{0}^{\prime}$ is not also insertible in each $D_{i}(1 \leq i \leq s)$. Therefore, by Lemma 3(1), we get

$$
e_{G}\left(\left\{w, z, u^{+}, v^{-}\right\}, D_{i}\right) \leq\left|D_{i}\right| \text { for } 1 \leq i \leq s .
$$


Since $P_{0}$ is maximal, it follows that

$$
e_{G}\left(\left\{w, z, u^{+}, v^{-}\right\}, H-P_{0}\right)=0 .
$$

By (5.1)-(5.5), we have

$$
\begin{aligned}
2 n+4 & \leq d_{G}(w)+d_{G}(z)+d_{G}\left(u^{+}\right)+d_{G}\left(v^{-}\right) \\
& \leq\left|Q_{0}\right|+\left|Q_{0}^{\prime}\right|+2 \times\left(\frac{|R|}{2}+1\right)+\sum_{i=1}^{s}\left|D_{i}\right| \leq|G|+2=2 n+2,
\end{aligned}
$$

a contradiction.

This contradiction shows that $d_{G\left[V\left(P_{0}\right)\right]}(x)+d_{G\left[V\left(P_{0}\right)\right]}(y) \leq \frac{\left|P_{0}\right|}{2}+1$, which, as explained in the first paragraph, completes the proof of Lemma 4.

Lemma 5 Let $k, n, G, M$ be the same as in Theorem 5. Under the same degree sum condition as Theorem [5, $G$ has an $M$-2-factor with exactly $k+1$ or exactly $k$ cycles of length at least 6 .

Proof of Lemma 5. Choose $k+1$ disjoint $M$-cycles $C_{1}, \ldots, C_{k+1}$ in $G$ so that

$$
\sum_{i=1}^{k+1}\left|C_{i}\right| \text { is as large as possible. }
$$

We may assume that $V(G) \backslash \bigcup_{i=1}^{k+1} V\left(C_{i}\right) \neq \emptyset$. Let $H=G-\bigcup_{i=1}^{k+1} V\left(C_{i}\right)$.

By (5.6), every edge in $M_{H}$ is not insertible in each $M$-cycle $C_{i}$, and hence by Lemma 3(1), $e_{G}\left(\{x, y\}, C_{i}\right) \leq \frac{\left|C_{i}\right|}{2}$ holds for $x y \in M_{H}$ and $1 \leq i \leq k+1$. Suppose that $H$ is not connected. Let $x_{1} y_{1}, x_{2} y_{2}$ be edges of $M$ in two different components of $H$ with orders $2 n_{1}, 2 n_{2}$ respectively, and let $n_{0}=\sum_{i=1}^{k+1} \frac{\left|C_{i}\right|}{2}$. Then $d_{G}\left(x_{h}\right)+d_{G}\left(y_{h}\right) \leq n_{0}+2 n_{h}(h \in\{1,2\})$, and so

$$
d_{G}\left(x_{1}\right)+d_{G}\left(y_{1}\right)+d_{G}\left(x_{2}\right)+d_{G}\left(y_{2}\right) \leq 2\left(n_{0}+n_{1}+n_{2}\right) \leq 2 n<2 n+4,
$$

contrary to hypothesis. Thus $H$ is connected.

Consider a maximal $M$-path $P_{0}[x, y]$ in $H$, and apply Lemma 4 with $s=k+1$ and $\left(D_{1}, \ldots, D_{s}, P_{0}\right)=\left(C_{1}, \ldots, C_{k+1}, P_{0}\right)$. If (iii) holds, then replacing $D_{i}$ by $D_{i}^{\prime}$ contradicts (5.6). If (iii) holds, then $e_{G}\left(\{x, y\}, C_{h}\right) \geq\left|C_{h}\right| / 2+1$ for some $h$ with $1 \leq h \leq k+1$, that is, $P_{0}$ is insertible in $C_{h}$ (by Lemma 3(1)), which contradicts (5.6) again. Thus (il) holds. Since $H$ is connected and $P_{0}$ is maximal, this implies that $H$ has an $M_{H}$-Hamilton cycle if $|H| \geq 4$.

By the degree sum condition, $G$ is connected and so $E_{G}\left(H, C_{i}\right) \neq \emptyset$ for some $i$ with $1 \leq i \leq$ $k+1$. We may assume that $i=1$. Since $H$ has an $M_{H}$-Hamilton cycle if $|H| \geq 4$, it follows that $G\left[V\left(H \cup C_{1}\right)\right]$ has an $M_{H \cup C_{1}}$-Hamilton path $P_{0}^{\prime}$. We now apply Lemma 4 with $s=k$ and $\left(D_{1}, \ldots, D_{s}, P_{0}\right)=\left(C_{2}, \ldots, C_{k+1}, P_{0}^{\prime}\right)$. Note that $\left|P_{0}^{\prime}\right| \geq 6$. If (ii) or (iii) holds, then this contradicts (5.6). Thus (iii) holds. This together with Lemma 3(1) implies $P_{0}^{\prime}$ is insertible in $C_{h}$ for some $h$ with $2 \leq h \leq k+1$. We name the $M_{P_{0}^{\prime} \cup C_{h}}$-Hamilton cycle of $G\left[V\left(P_{0}^{\prime} \cup C_{h}\right)\right]$ as $C_{h}^{\prime}$, and then $C_{2} \cup \cdots \cup C_{h-1} \cup C_{h}^{\prime} \cup C_{h+1} \cup \cdots \cup C_{k+1}$ forms an $M$-2-factor with exactly $k$ cycles of length at least 6 .

Now we are ready to prove Theorem 5 . 
Proof of Theorem 5. By Lemma 5, we may assume that $G$ has an $M$-2-factor with exactly $k+1$ cycles $C_{1}, \ldots, C_{k+1}$ of length at least 6 , but $G$ has no $M$-2-factor with exactly $k$ cycles of length at least 6 (since otherwise, the result holds). Then, the following fact holds.

Fact 5.1 Let $I \subseteq\{1,2, \ldots, k+1\}$ with $|I| \geq 2$, and let $C=\bigcup_{i \in I} C_{i}$. Then $G[V(C)]$ does not have an $M_{C}$-2-factor with exactly $|I|-1$ cycles of length at least 6 .

Let $X$ and $Y$ be partite sets of $G$, and we may assume that $x x^{+} \in E\left(C_{i}\right) \cap M$ for each cycle $C_{i}$ and $x \in V\left(C_{i}\right) \cap X$.

Claim 5.2 For any two cycles $C_{i}$ and $C_{j}$ with $i \neq j$, there exist no four vertices $x_{i} \in V\left(C_{i}\right) \cap X$, $x_{j} \in V\left(C_{j}\right) \cap X, y_{i} \in V\left(C_{i}\right) \cap Y$ and $y_{j} \in V\left(C_{j}\right) \cap Y$ such that $\left\{x_{i} y_{j}, y_{i} x_{j}\right\} \subseteq E(G)$.

Proof. Suppose that there exist two cycles and four vertices as specified in the claim. We may assume that $i=1$ and $j=2$. Choose $C_{1}, C_{2}, x_{1}, y_{1}, x_{2}$ and $y_{2}$ so that

$$
x_{1} y_{1} \in E\left(C_{1}\right) \backslash M \text { or } x_{2} y_{2} \in E\left(C_{2}\right) \backslash M \text { if possible. }
$$
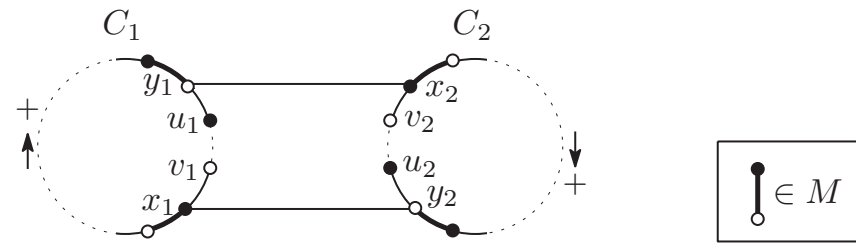

Figure 5: The vertices $x_{h}, y_{h}, u_{h}, v_{h}(h \in\{1,2\})$

Let $u_{1}=y_{1}^{+}, v_{1}=x_{1}^{-}, u_{2}=y_{2}^{+}$and $v_{2}=x_{2}^{-}$(see Figure 5). We consider the $M_{C_{1} \cup C_{2}}$ ) Hamilton paths $P_{0}=u_{1} \overrightarrow{C_{1}} y_{1} x_{2} \overrightarrow{C_{2}} v_{2}$ and $P_{0}^{\prime}=v_{1} \overleftarrow{C_{1}} x_{1} y_{2} \overleftarrow{C_{2}} u_{2}$ in $G\left[V\left(C_{1} \cup C_{2}\right)\right]$ and we apply Lemma 4 with $s=k-1,\left(D_{1}, \ldots, D_{s}, P_{0}\right)=\left(C_{3}, \ldots, C_{k+1}, P_{0}\right)$ and $\left(D_{1}, \ldots, D_{s}, P_{0}\right)=$ $\left(C_{3}, \ldots, C_{k+1}, P_{0}^{\prime}\right)$, respectively. Then, either (ii), (iii) or (iii) holds for $\left(C_{3}, \ldots, C_{k+1}, P_{0}\right)$ and $\left(C_{3}, \ldots, C_{k+1}, P_{0}^{\prime}\right)$, respectively. Combining this with Fact 5.1, we see that (iii) holds for each case. Let $H_{12}=G-V\left(C_{1} \cup C_{2}\right)$. Then, we get

$$
\begin{aligned}
e_{G}\left(\left\{u_{1}, v_{1}\right\}, H_{12}\right)+e_{G}\left(\left\{u_{2}, v_{2}\right\}, H_{12}\right) & =e_{G}\left(\left\{u_{1}, v_{2}\right\}, H_{12}\right)+e_{G}\left(\left\{v_{1}, u_{2}\right\}, H_{12}\right) \\
& \geq\left(\frac{\left|H_{12}\right|}{2}+1\right)+\left(\frac{\left|H_{12}\right|}{2}+1\right)=\left|H_{12}\right|+2 .
\end{aligned}
$$

This implies that

$$
e_{G}\left(\left\{u_{1}, v_{1}\right\}, H_{12}\right) \geq \frac{\left|H_{12}\right|}{2}+1 \text { or } e_{G}\left(\left\{u_{2}, v_{2}\right\}, H_{12}\right) \geq \frac{\left|H_{12}\right|}{2}+1 .
$$

Without loss of generality, we may assume that $e_{G}\left(\left\{u_{1}, v_{1}\right\}, H_{12}\right) \geq \frac{\left|H_{12}\right|}{2}+1$. Then there exists a cycle $C_{h}$ with $3 \leq h \leq k+1$ such that $e_{G}\left(\left\{u_{1}, v_{1}\right\}, C_{h}\right) \geq \frac{\left|C_{h}\right|}{2}+1$. Hence by Lemma (3)(1), there is an insertion edge $b_{h} b_{h}^{+}$of $u_{1} \overrightarrow{C_{1}} v_{1}$ in $C_{h}$ such that $u_{1} b_{h}, v_{1} b_{h}^{+} \in E(G)$. This also implies 
that $x_{1} y_{1} \in E\left(C_{1}\right) \backslash M$ or $x_{2} y_{2} \in E\left(C_{2}\right) \backslash M$ (since otherwise, replacing $\left(C_{1}, C_{2}, x_{1}, y_{1}, x_{2}, y_{2}\right)$ with $\left(C_{1}, C_{h}, u_{1}, v_{1}, b_{h}^{+}, b_{h}\right)$, this contradicts the choice (5.7)).

If $x_{1} y_{1} \in E\left(C_{1}\right) \backslash M$, that is, $u_{1}=x_{1}$, then $x_{1} \overrightarrow{C_{1}} y_{1}$ is insertible in $C_{h}$, which contradicts Fact 5.1 (see the left figure of Figure [6).
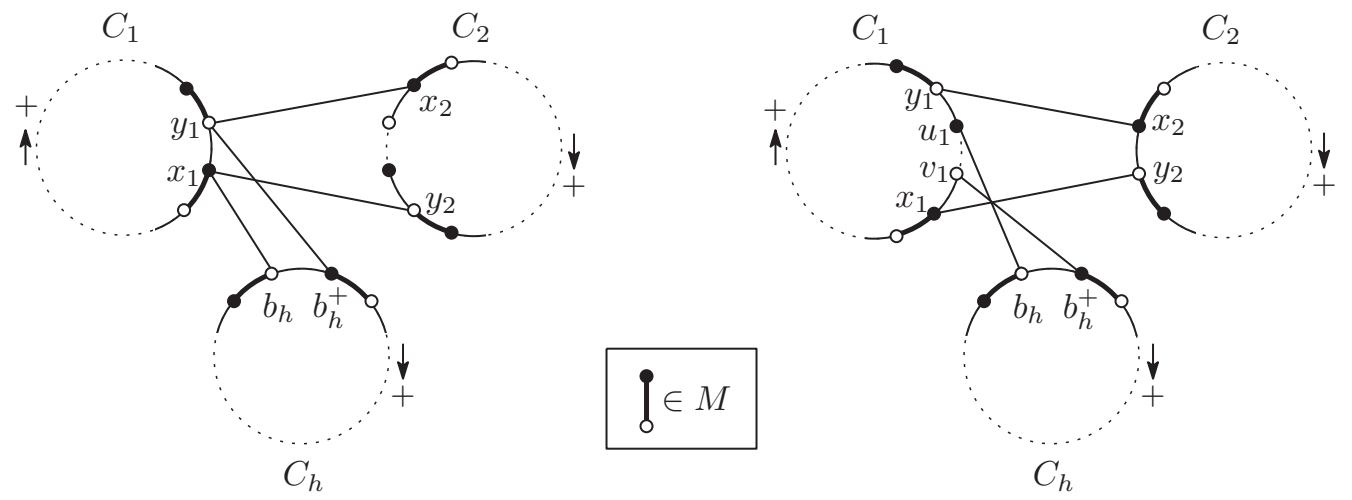

Figure 6: Two cases for Claim 5.2

Thus, $x_{1} y_{1} \notin E\left(C_{1}\right) \backslash M$, and hence $x_{2} y_{2} \in E\left(C_{2}\right) \backslash M$, that is, $u_{2}=x_{2}$. Then, $x_{1} \overrightarrow{C_{1}} y_{1} x_{2} \overrightarrow{C_{2}} y_{2} x_{1}$ and $u_{1} \overrightarrow{C_{1}} v_{1} b_{h}^{+} \overrightarrow{C_{h}} b_{h} u_{1}$ form an $M_{C_{1} \cup C_{2} \cup C_{h}}$-2-factor with two cycles of length at least 6 in $G\left[V\left(C_{1} \cup\right.\right.$ $\left.C_{2} \cup C_{h}\right)$ ], which contradicts Fact 5.1 (see the right figure of Figure 6).

Since $2 n>6(k+1)$, we may assume that $\left|C_{1}\right| \geq 8$. Note that $E_{G}\left(C_{1}, G-C_{1}\right) \neq \emptyset$ because $\sigma_{1,1}(G) \geq n+2$ implies $G$ is connected and $k+1 \geq 2$, and hence we may also assume that there exist two vertices $y_{1} \in V\left(C_{1}\right) \cap Y$ and $x_{2} \in V\left(C_{2}\right) \cap X$ such that $y_{1} x_{2} \in E(G)$. Let $x_{1} \in V\left(C_{1}\right)$ and $y_{2} \in V\left(C_{2}\right)$ with $x_{1} y_{1} \in E\left(C_{1}\right) \cap M$ and $x_{2} y_{2} \in E\left(C_{2}\right) \cap M$ (i.e., $x_{1}=y_{1}^{-}$and $y_{2}=x_{2}^{+}$). Let $u_{1}=y_{1}^{+}, v_{1}=x_{1}^{-}$and $v_{2}=x_{2}^{-}$.

By Claim 5.2, $E_{G}\left(x_{1}, C_{2}\right)=E_{G}\left(v_{2}, C_{1}\right)=\emptyset$. In particular, $x_{1} v_{2} \notin E(G)$. Let $H_{12}=$ $G-V\left(C_{1} \cup C_{2}\right)$. Then we obtain

$$
\begin{aligned}
e_{G}\left(\left\{x_{1}, v_{2}\right\}, H_{12}\right) & =\left(d_{G}\left(x_{1}\right)+d_{G}\left(v_{2}\right)\right)-d_{G\left[V\left(C_{1}\right)\right]}\left(x_{1}\right)-e_{G}\left(x_{1}, C_{2}\right)-e_{G}\left(v_{2}, C_{1}\right)-d_{G\left[V\left(C_{2}\right)\right]}\left(v_{2}\right) \\
& \geq(n+2)-\left|C_{1}\right| / 2-0-0-\left|C_{2}\right| / 2=\left|H_{12}\right| / 2+2 .
\end{aligned}
$$

Therefore $k \geq 2$ and there exists a cycle $C_{h}$ with $3 \leq h \leq k+1$ such that $e_{G}\left(\left\{x_{1}, v_{2}\right\}, C_{h}\right) \geq$ $\left|C_{h}\right| / 2+1$. Without loss of generality, we may assume that $h=3$. Then by Lemma 3(1), there exists an edge $y_{3} u_{3}$ with $u_{3} \in V\left(C_{3}\right) \cap X$ and $y_{3} \in V\left(C_{3}\right) \cap Y$ in $E\left(C_{3}\right) \backslash M$ such that

$$
\left\{x_{1} y_{3}, v_{2} u_{3}\right\} \subseteq E(G)
$$

(see Figure 7).

Now consider the $M$-path $P_{0}=u_{1} \overrightarrow{C_{1}} v_{1}$ and the $M$-cycle $D_{0}=x_{1} y_{1} x_{2} \overrightarrow{C_{2}} v_{2} u_{3} \overrightarrow{C_{3}} y_{3} x_{1}$ in $G\left[V\left(C_{1} \cup C_{2} \cup C_{3}\right)\right]$. Recall that $\left|C_{1}\right| \geq 8$, that is, $\left|P_{0}\right| \geq 6$. We apply Lemma 4 with $s=$ $k-1$ and $\left(D_{1}, \ldots, D_{s}, P_{0}\right)=\left(D_{0}, C_{4}, \ldots, C_{k+1}, P_{0}\right)$. Then, either (ii), (iii) or (iii) holds for $\left(D_{0}, C_{4}, \ldots, C_{k+1}, P_{0}\right)$. Combining this with Fact [5.1, we see that (iii) holds. Let $H_{0}=G-P_{0}$. 


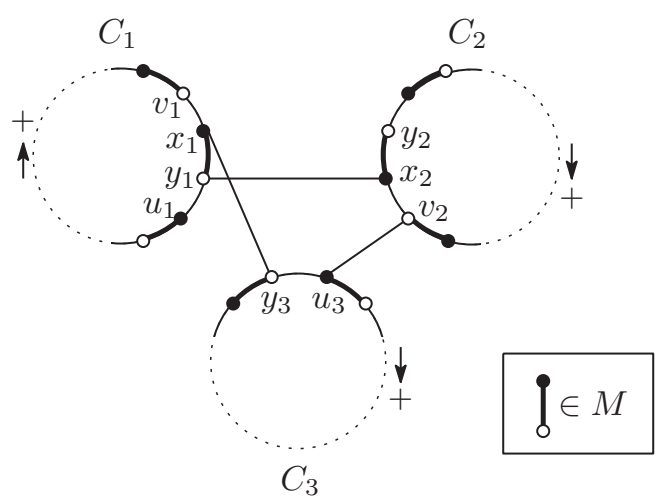

Figure 7: $\left\{x_{1} y_{3}, v_{2} u_{3}\right\} \subseteq E(G)$

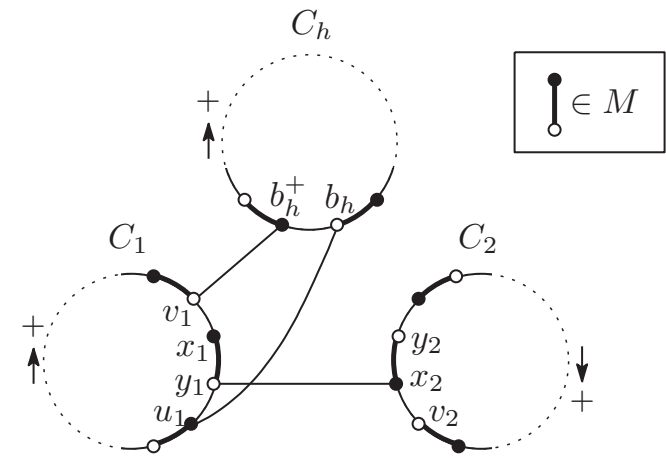

Figure 8: $e_{G}\left(\left\{u_{1}, v_{1}\right\}, C_{h}\right) \geq \frac{\left|C_{h}\right|}{2}+1$

Then we get $e_{G}\left(\left\{u_{1}, v_{1}\right\}, H_{0}\right) \geq\left|H_{0}\right| / 2+1$. Hence either (a) there exists a cycle $C_{h}$ with $4 \leq h \leq k$ such that $e_{G}\left(\left\{u_{1}, v_{1}\right\}, C_{h}\right) \geq \frac{\left|C_{h}\right|}{2}+1$, or (b) $e_{G}\left(\left\{u_{1}, v_{1}\right\}, D_{0}\right) \geq \frac{\left|D_{0}\right|}{2}+1$. If (a) holds, then by Lemma 3(1), there exists an edge $b_{h} b_{h}^{+}$in $E\left(C_{h}\right)$ such that $u_{1} b_{h}, v_{1} b_{h}^{+} \in E(G)$, which contradicts Claim 5.2 (see Figure 8). Thus $e_{G}\left(\left\{u_{1}, v_{1}\right\}, D_{0}\right) \geq \frac{\left|D_{0}\right|}{2}+1$. Since $x_{1} y_{3} \in E_{G}\left(C_{1}, C_{3}\right)$ and $y_{1} x_{2} \in E_{G}\left(C_{1}, C_{2}\right)$, it follows from Claim 5.2 that $E_{G}\left(v_{1}, C_{3}\right)=\emptyset$ and $E_{G}\left(u_{1}, C_{2}\right)=\emptyset$. Therefore, the inequality $e_{G}\left(\left\{u_{1}, v_{1}\right\}, D_{0}\right) \geq \frac{\left|D_{0}\right|}{2}+1$ implies that

$$
V\left(C_{3}\right) \cap Y \subseteq N_{G}\left(u_{1}\right) \text { and } V\left(C_{2}\right) \cap X \subseteq N_{G}\left(v_{1}\right) .
$$

Let $x_{3} \in V\left(C_{3}\right)$ with $x_{3} y_{3} \in E\left(C_{3}\right) \cap M$.

Claim 5.3 $E_{G}\left(x_{3}, C_{2}-\left\{v_{2}\right\}\right)=\emptyset$.

Proof. Suppose that there exists a vertex $b_{2} \in V\left(C_{2}\right) \backslash\left\{v_{2}\right\}$ with $x_{3} b_{2} \in E(G)$. Recall that by (5.8), $x_{1} y_{3}, v_{2} u_{3} \in E(G)$. Recall also that by (5.9), $u_{1} x_{3}^{-}, v_{1} b_{2}^{+} \in E(G)$. Then $u_{1} \overrightarrow{C_{1}} v_{1} b_{2}^{+} \overrightarrow{C_{2}} v_{2} u_{3} \overrightarrow{C_{3}} x_{3}^{-} u_{1}$ and $x_{1} y_{1} x_{2} \overrightarrow{C_{2}} b_{2} x_{3} y_{3} x_{1}$ form an $M_{C_{1} \cup C_{2} \cup C_{3}}$-2-factor with two cycles of length at least 6 in $G\left[V\left(C_{1} \cup C_{2} \cup C_{3}\right)\right]$, which contradicts Fact 5.1 (see Figure 91).

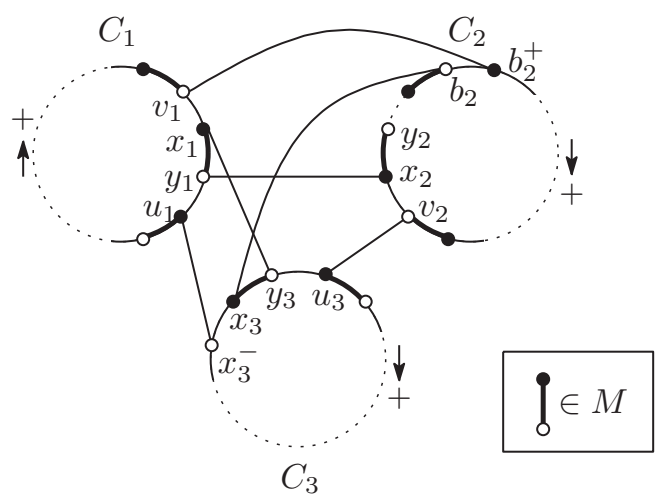

Figure 9: Claim 5.3

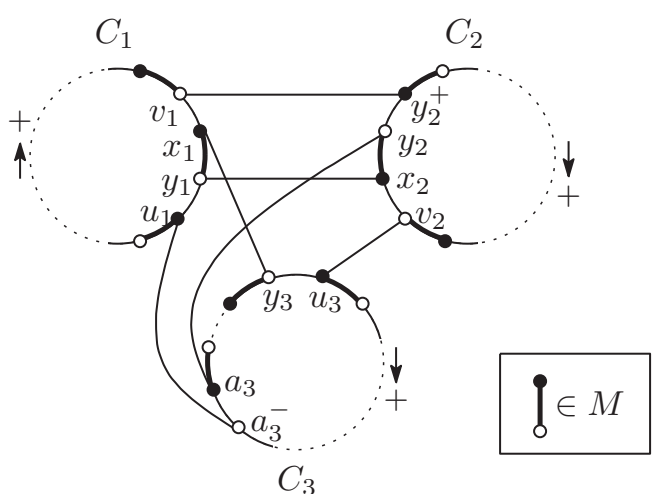

Figure 10: Claim 5.4

Claim 5.4 $E_{G}\left(y_{2}, C_{3}-\left\{u_{3}\right\}\right)=\emptyset$. 
Proof. Suppose that there exists a vertex $a_{3} \in V\left(C_{3}\right) \backslash\left\{u_{3}\right\}$ with $y_{2} a_{3} \in E(G)$. Recall that by (15.8), $x_{1} y_{3}, v_{2} u_{3} \in E(G)$. Recall also that by (15.9), $u_{1} a_{3}^{-}, v_{1} y_{2}^{+} \in E(G)$. Then $u_{1} \overrightarrow{C_{1}} v_{1} y_{2}^{+} \overrightarrow{C_{2}} v_{2} u_{3} \overrightarrow{C_{3}} a_{3}^{-} u_{1}$ and $x_{1} y_{1} x_{2} y_{2} a_{3} \overrightarrow{C_{3}} y_{3} x_{1}$ form an $M_{C_{1} \cup C_{2} \cup C_{3}}$-2-factor with two cycles of length at least 6 in $G\left[V\left(C_{1} \cup C_{2} \cup C_{3}\right)\right]$, which contradicts Fact 5.1 (see Figure 10).

Let $H_{23}=G-V\left(C_{2} \cup C_{3}\right)$. Since $v_{2} u_{3} \in E_{G}\left(C_{2}, C_{3}\right)$, it follows from Claim 5.2 that $x_{2} y_{3} \notin E(G)$ and

$$
\begin{aligned}
e_{G}\left(\left\{x_{2}, y_{3}\right\}, H_{23}\right) & =\left(d_{G}\left(x_{2}\right)+d_{G}\left(y_{3}\right)\right)-d_{G\left[V\left(C_{2}\right)\right]}\left(x_{2}\right)-e_{G}\left(x_{2}, C_{3}\right)-e_{G}\left(y_{3}, C_{2}\right)-d_{G\left[V\left(C_{3}\right)\right]}\left(y_{3}\right) \\
& \geq(n+2)-\left|C_{2}\right| / 2-0-0-\left|C_{3}\right| / 2=\left|H_{23}\right| / 2+2 .
\end{aligned}
$$

On the other hand, by Claims 5.3 and [5.4, we have $x_{3} y_{2} \notin E(G)$ and

$$
\begin{aligned}
e_{G}\left(\left\{x_{3}, y_{2}\right\}, H_{23}\right) & =\left(d_{G}\left(x_{3}\right)+d_{G}\left(y_{2}\right)\right)-e_{G}\left(x_{3}, C_{2}\right)-d_{G\left[V\left(C_{3}\right)\right]}\left(x_{3}\right)-d_{G\left[V\left(C_{2}\right)\right]}\left(y_{2}\right)-e_{G}\left(y_{2}, C_{3}\right) \\
& \geq(n+2)-1-\left|C_{3}\right| / 2-\left|C_{2}\right| / 2-1=\left|H_{23}\right| / 2 .
\end{aligned}
$$

Therefore we deduce

$$
e_{G}\left(\left\{x_{2}, y_{2}, x_{3}, y_{3}\right\}, H_{23}\right) \geq\left|H_{23}\right|+2 \text {. }
$$

This implies that

$$
e_{G}\left(\left\{x_{2}, y_{2}\right\}, H_{23}\right) \geq \frac{\left|H_{23}\right|}{2}+1 \text { or } e_{G}\left(\left\{x_{3}, y_{3}\right\}, H_{23}\right) \geq \frac{\left|H_{23}\right|}{2}+1 .
$$

Without loss of generality, we may assume that $e_{G}\left(\left\{x_{2}, y_{2}\right\}, H_{23}\right) \geq \frac{\left|H_{23}\right|}{2}+1$. Then there exists a cycle $C_{h}$ with $h=1$ or $4 \leq h \leq k+1$ such that $e_{G}\left(\left\{x_{2}, y_{2}\right\}, C_{h}\right) \geq \frac{\left|C_{h}\right|}{2}+1$. But this clearly leads to a contradiction with Claim [5.2, and this completes the proof of Theorem 5 .

\section{Related problems}

To conclude the paper, we mention related problems. In order to prove our main result (Theorem 1), we have considered $k$ disjoint $M$-cycles of length at least 6 in bipartite graphs (Step 1) and have given Theorem 4 in Section 3. However, in the proof of Theorem 1, we do not use "each $M$-cycle has length at most 8" in the conclusion of Theorem 4 (see the proof in Section 3). Therefore, one may consider the problem whether the degree condition can be weakened if we drop the condition "length at most 8" in Theorem 4. Note that a much weaker degree condition than the one of Theorem B guarantees the existence of $k$ disjoint cycles in simple undirected graphs.

Theorem E (Enomoto [6], Wang [14]) Let $k$ be a positive integer, and let $G$ be a graph of order at least $3 k$. If $\sigma_{2}(G) \geq 4 k-1$, then $G$ contains $k$ disjoint cycles.

Considering the relation between simple undirected graphs, digraphs and bipartite graphs (Remarks 1 and 2), we can consider the following problem as a digraph version of Theorem E 
Problem 6 Let $k$ be a positive integer, and let $D$ be a digraph of order $n \geq 3 k$. Is it true that, if $d_{D}^{+}(u)+d_{D}^{-}(v) \geq 4 k-1$ for every two distinct vertices $u$ and $v$ with $(u, v) \notin A(D)$, then $D$ contains $k$ disjoint directed cycles of length at least 3 ?

As another related problem, the following "minimum out-degree" condition is conjectured by Bermond and Thomassen (1981) for the existence of just $k$ disjoint directed cycles.

Conjecture F (Bermond, Thomassen [3]) Let $k$ be a positive integer, and let $D$ be a digraph. If every vertex has out-degree at least $2 k-1$, then $D$ contains $k$ disjoint directed cycles.

The case $k=1$ of this conjecture is an easy problem, and the cases $k=2$ and $k=3$ are proved in [12] and [10, respectively. Alon [1] proved that the conclusion holds if every vertex has out-degree at least $64 k$, but the conjecture as stated remains open.

\section{References}

[1] N. Alon, Disjoint directed cycles, J. Combin. Theory Ser. B 68 (1996) 167-178.

[2] D. Amar, E. Flandrin, G. Gancarzewicz, A. Paweł Wojda, Bipartite graphs with every matching in a cycle, Discrete Math. 307 (2007) 1525-1537.

[3] J.C. Bermond, C. Thomassen, Cycles in digraphs-A survey, J. Graph Theory 5 (1981) $1-43$.

[4] S. Brandt, G. Chen, R.J. Faudree, R.J. Gould, L. Lesniak, Degree conditions for 2-factors, J. Graph Theory 24 (1997) 165-173.

[5] R. Diestel, Graph Theory, Fourth edition. Graduate Texts in Mathematics, 173, Springer, Heidelberg, 2010.

[6] H. Enomoto, On the existence of disjoint cycles in a graph, Combinatorica 18 (1998) 487492.

[7] R.J. Gould, A look at cycles containing specified elements of a graph, Discrete Math. 309 (2009) 6299-6311.

[8] M. Las Vergnas, Ph.D. Thesis, University of Paris, 1972.

[9] H. Li, Generalizations of Dirac's theorem in Hamiltonian graph theory-A survey, Discrete Math. 313 (2013) 2034-2053.

[10] N. Lichiardopol, At. Pór, J.S. Sereni, A step toward the Bermond-Thomassen conjecture about disjoint cycles in digraphs, SIAM J. Discrete Math 23 (2009) 979-992.

[11] O. Ore, Note on Hamilton circuits, Amer. Math. Monthly 67 (1960) 55.

[12] C. Thomassen, Disjoint cycles in digraphs, Combinatorica 3 (1983) 393-396. 
[13] W.T. Tutte, A short proof of the factor theorem for finite graphs, Canadian J. Math. 6 (1954) 347-352.

[14] H. Wang, On the maximum number of independent cycles in a graph, Discrete Math. 205 (1999) 183-190.

[15] D.R. Woodall, Sufficient conditions for cycles in digraphs, Proc. Lond. Math. Soc. 24 (1972) 739-755.

[16] Z.B. Zhang, X. Zhang, X. Wen, Directed Hamilton cycles in digraphs and matching alternating Hamilton cycles in bipartite graphs, SIAM J. Discrete Math. 27 (2013) 274-289. 